Radial and Nonradial Pulsations as Probes of Stellar Physics

ASP Conference Series, Vol. 259, 2002

C. Aerts, T.R. Bedding, \& J. Christensen-Dalsgaard, eds.

\title{
Rapid Light Variations of the Bright Be Star $\phi$ Per
}

\author{
D. Sudar, D. Ruždjak \\ Hvar Observatory, Faculty of Geodesy, Zagreb University, Kačićeva 26, \\ 10000 Zagreb, Croatia
}

\begin{abstract}
An analysis of the $U B V$ photometric measurements obtained at Hvar Observatory and Hp HIPPARCOS photometry has been performed in order to disclose the suspected rapid periodic variations of the Be star $\phi$ Per. The reliability of the derived 0.5 day period is examined and the difficulties of detecting the period due to the complex character of the light variations on the longer time-scales are outlined. Possible explanations of these rapid variations are briefly discussed.
\end{abstract}

\section{Introduction}

$\phi$ Per (HD 10516, HR 496) is a well known spectroscopic Be binary, with a 126.7-day orbital period, which consists of a B0.5IV primary and a compact secondary (Gies et al., 1998). Its complex photometric behaviour including the long-term, phase locked and rapid light variations are described in Božić et al. (1995). For the rapid variations they disclosed a formal 0.66-day period while from the HIPPARCOS Hp photometry Hubert \& Floquet (1998) found the value of 0.470 days.

\section{Analysis}

We analysed the homogenous set of 373 individual $U B V$ measurements secured from 2444935 to $2451943 \mathrm{HJD}$ at Hvar observatory and compared them with HIPPARCOS Hp magnitudes transformed to Johnson $B V$ filters.

Due to the suspected period of nearly 0.5 day one observes the star in nearly the same phase during the whole observing season. Consequently, smoothing through observing seasons destroys the rapid light variations curve. For those reasons we analysed rapid light variations without seasonal smoothing. The Fourier Transform (FT) analysis of Hvar V measurements revealed the period of 0.49926 days. One is suspicious of a period close to 0.5 days because of the strong alliasing problem connected with ground-based observations. HIPPARCOS measurements are not affected by this problem and the reported period confirms the Hvar result.

Of special interest is a series of 20 measurements obtained by HIPPARCOS in the interval 2448167-2448169 HJD. FT analysis of those measurements reveals a period of 0.49202 days. This is in agreement with the period obtained with Hvar measurements. 


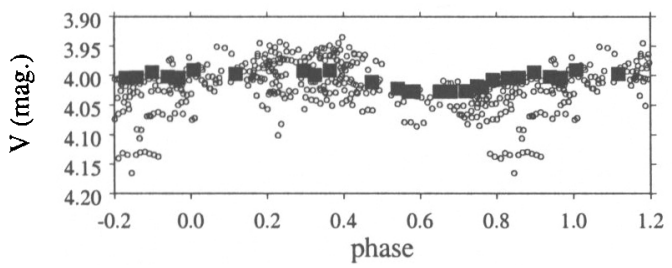

Figure 1. Comparison of Hvar (open circles) and 20 HIPPARCOS measurements (filled squares), phased to a frequency of $0.49926 \mathrm{c} \mathrm{d}^{-1}$.

It can be seen that the HIPPARCOS series of measurements is also in phase with Hvar observations. The large scatter of the Hvar measurements is the result of long-term and phase locked variations that were not removed. It is possible that the amplitude is varying but multiperiodicity cannot be completely excluded.

A rotational model for Be stars (RM) predicts that the photometric period is equal to rotational period of the star. The critical periods for the parameters published by Gies et al. (1998) and Božić et al. (1995), calculated from Harmanec's (1988) calibration and for $i>63^{\circ}$, are in the range $0.357-0.783 \mathrm{c} \mathrm{d}^{-1}$. Although for some parameters the critical period is shorter than the observed period, it seems that the photometric period is not the rotational period of $\phi$ Per. The first argument is that Be stars rotate roughly with $70 \%$ of critical velocity and the second argument is that fast rotating stars are elongated on the equator so one should use the equatorial radius, which is much closer to $R_{E}=1.5 R_{\text {Polar }}$. If the double photometric period is the rotation period (double wave curve), RM would require a symmetric distribution of spots around the rotation axis.

The NRP model is capable of explaining the observed photometric period, although accurate identification of the mode is not possible. A restriction is that $|m|<4$ because otherwise the light curve would be smeared due to the fast rotation (Balona, 1995). At present there is no spectroscopic confirmation for rapid variability in $\phi$ Per. More spectroscopic measurements of good quality are required to confirm and further investigate the possibility of NRP in $\phi$ Per.

\section{References}

Balona, L. A. 1995, MNRAS, 277, 1547

Božić, H., Harmanec, P., Horn, J., Koubsky, P., Scholz, G., McDavid, D., Hubert, A.M., \& Hubert, H. 1995, A\&A, 304, 235

Gies, D.R., Bagnuolo, W.G. Jr., Ferrara, E.C., Kaye, A.B., Thaller, M.L., Penny, L.R., \& Peters, G.J. 1998, ApJ, 493, 440

Harmanec, P. 1988, Bull. Astron. Inst. Czechosl., 39, 329

Hubert, A.M. \& Floquet, M. 1998, A\&A, 335, 565 


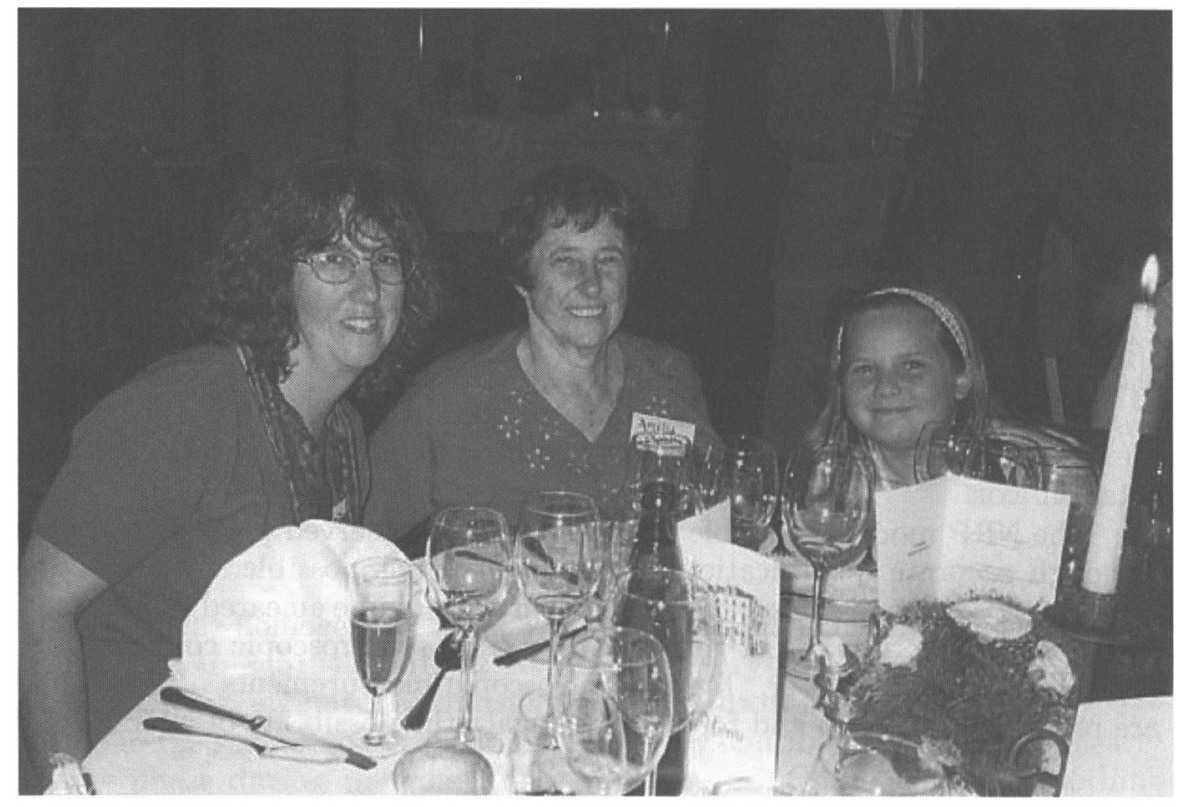

Amelia Wehlau brought her daughter and granddaughter to Leuven and it seems that they are all having a good time. 